\title{
Energy model of electric drive of centrifugal borehole pump
}

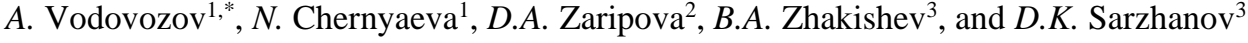 \\ ${ }^{1}$ Vologda State University, Vologda, Russia \\ ${ }^{2}$ Kazan State Power Engineering University, Kazan, Russia \\ ${ }^{3}$ L.N. Gumilyov Eurasian National University, Astana, Kazakhstan
}

\begin{abstract}
A mathematical model of an electric drive of a centrifugal borehole pump is proposed for evaluating its energy consumption in operating conditions. The model is based on the use of minimum set of technical parameters of the pump, presented in the public data of the manufacturer. The correctness of the calculated formulas is confirmed by experimental data provided in the program for equipment search and selection from Grundfos Product Center. Based on the created model, a comparative analysis of the energy efficiency of products from various manufacturers was made. It is proved that when choosing a centrifugal borehole pump of the local water supply system, it is necessary to pay attention to the energy efficiency indicators of electric drive in order to make a final decision.
\end{abstract}

\section{Introduction}

The use of groundwater sources on the basis of wells is currently a common technical solution when designing industrial and individual water supply systems. In this case water supply system is based on the application of a borehole pump with an electric drive and an automation unit which controls the object with specified quality indicators [1-3].

To equip the water wells, the manufacturers offer a whole range of electric centrifugal pumps with a wide range of prices and technical characteristics. At the same time, pumps of all manufacturers, despite the development and widespread use of innovative technologies, still have relatively low efficiencies and high specific energy consumption [4-6]. The energy consumed by electric drives of centrifugal pumps is spent not only on the rise of water, but also on the losses in the mechanism and in the submersible electric motor. If we consider that up to $85 \%$ of the cost of operating any life-support system is the cost of electricity, it becomes clear why, when choosing a well pump in the process of designing electrical equipment for wells, greater attention is paid to energy consumption $[7,8]$.

The Russian market of pumping equipment can be divided into two characteristic groups. Products of international manufacturers, such as Grundfos (Denmark), Pedrollo, Aquario (Italy), Wilo (Germany), have higher price, but at the same time their specifications are accompanied by detailed descriptions, characteristics, selection and calculation methods, examples of ready-made solutions that promote products to the consumer [4-6]. The Russian manufacturers and firms from neighboring countries, for example, Unipump, Jeelex (Russia), Belamos (Belarus), Promelectro (Ukraine), as a rule, offer lower-cost solutions. In this case, technical documentation presented in free Internet data, unfortunately, does not contain all the necessary information to assess the economic and energy efficiency of the project. The list of technical characteristics of borehole pumps usually indicates the minimum set of parameters: nominal head, nominal flow rate and nominal power consumption. Often, even the pressure characteristics of the pump are not freely available. Recommendations on the choice of equipment in this case are reduced to the methods of selecting a pump for the nominal head and nominal flow rate, which must be agreed with the consumer $[9,10]$.

This article proposes a mathematical model of an electric centrifugal borehole pump for calculating its energy efficiency, based on the use of minimum set of parameters presented in the manufacturer's public data.

\section{Materials and methods}

The specific energy consumption is taken as the main indicator of the energy efficiency of a well pump, i.e. the cost of electrical energy for the supply of 1 cubic meter of water:

$$
E=P / Q
$$

where: $E$ is specific energy consumption, $\mathrm{kW} \mathrm{h} / \mathrm{m}^{3}, P$ is power consumed by pump electric drive, $\mathrm{kW}, Q$ is pump consumption, $\mathrm{m}^{3} / \mathrm{h}$.

Since the electric power consumed by the pump electric drive is determined by its payload, in this particular case, it can be calculated based on the hydraulic power $P_{g}(\mathrm{~kW})$ at the output of the pump unit and its coefficient of efficiency (COE) $\eta$, measured in $\%$, is:

\footnotetext{
* Corresponding author: am.vodovozov@gmail.com
} 


$$
P=100 P_{g} / Q
$$

The hydraulic power of pump is determined by the generated head $H$ and capacity $Q$ [11]:

$$
P=10^{-6} \mathrm{mgQH} / 3.6
$$

where: $m g=9800$ is specific weight of water, $\mathrm{N} / \mathrm{m}^{3}, H$ is pump head, $\mathrm{m}$.

In general case, the pump coefficient of efficiency, its flow rate and head depend on the operating point on its characteristics: pressure characteristics and $\mathrm{COE}$ (Figure 1).

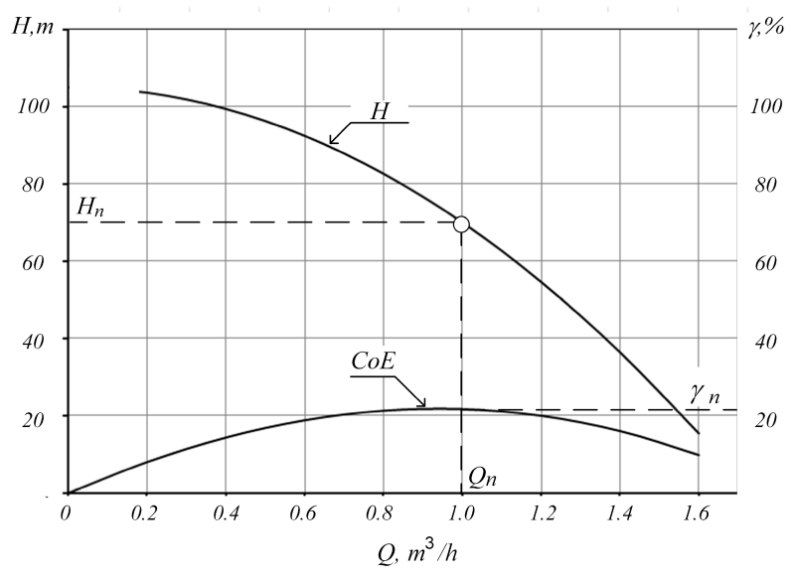

Fig. 1. Example of centrifugal borehole pump performance data (Grundfoss SP1A-18 pump characteristics [11]).

A nominal operating point is set on the pressure characteristic $H(Q)$ with nominal flow rate $Q_{n}$, nominal head $\mathrm{H}_{\mathrm{n}}$, and in which the COE has a nominal value $\eta_{n}$, close to the maximum. For practical calculations and simulations the pressure characteristics in graphical form (Figure 1) is unacceptable. To describe it, we will use a second-order equation, the coefficients of which are selected on the basis of experimental data. We use the most general formula proposed in [12]:

$$
H=H_{\max }\left[1-k\left(Q / Q_{n}\right)^{2}\right]
$$

where $H_{\max }$ is maximum head; $m, k$ is coefficient of proportionality.

The proportionality coefficient $\mathrm{k}$ is determined from the condition: $H=H_{n}$, at $Q=Q_{n}$. In this case, $k=(1-$ $\left.H_{n} / H_{\max }\right)$. Some manufacturers specify the maximum head in the passport data of the pump, or it can be determined from the head characteristics, as head at $Q=0$. In the absence of this information, for approximate calculations, $H_{\max }=1.5 H_{n}$ can be taken, which is true, for example, for Grundfoss SP series centrifugal borehole pumps [11].

The nominal efficiency of pump $\eta_{n}$ can be determined from its passport characteristics

$$
\eta_{n}=100 P_{g n} / P_{n}
$$

where $P_{n}$ is rated power consumption of pump, kW; $P_{g n}$ is hydraulic power of pump in nominal passport mode:

$$
P_{g n}=10^{-6} m g Q_{n} H_{n} / 3.6
$$

The maximum pump efficiency corresponds to a point with a nominal consumption. Mathematical descriptions of the dependence $\eta(Q)$ are different. In [13], a description was proposed in the form of a parabola.

$$
\eta=\eta_{n}\left[2 Q / Q_{n}-\left(Q / Q_{n}\right)^{2}\right]
$$

As a result, we have a system of equations (1-7), which allows one to calculate the specific energy consumption of pump $\mathrm{E}\left(\mathrm{kW} \mathrm{h} / \mathrm{m}^{3}\right)$ in a given operation mode.

As an example, we will perform energy efficiency calculations for a number of Grundfoss series SP1A pumps under the condition of providing a head at the outlet of the pump $H=60 \mathrm{~m}$. Such a head allows one to raise water from the dynamic level of the well $(30 \mathrm{~m})$ and ensure comfortable pressure in the water supply system (3 atm). The results of the calculations are shown in Table 1. The initial data for the calculations are taken from the Grundfoss Product Center (GPC) borehole pump selection program [11]. For comparison, the table shows data on the energy efficiency of pumps obtained from the GPC system under the same initial conditions.

Table 1. The results of energy efficiency calculations for pumps of the SP series by Grundfoss.

\begin{tabular}{|c|c|c|c|c|c|}
\hline \multicolumn{5}{|c|}{ Data from GPC } & Calculation \\
\hline Type & $\begin{array}{c}P_{n}, \\
\mathrm{~kW}\end{array}$ & $\begin{array}{c}Q_{n} \\
\mathrm{~m}^{3} / \mathrm{h}\end{array}$ & $\begin{array}{c}H_{n}, \\
\mathrm{~m}\end{array}$ & $\begin{array}{c}\mathrm{E}, \\
\mathrm{kW} \mathrm{h} / \mathrm{m}^{3}\end{array}$ & $\begin{array}{c}\mathrm{E}, \\
\mathrm{kW} \mathrm{h} / \mathrm{m}^{3}\end{array}$ \\
\hline SP1A-14 & 0.65 & 1 & 53 & 0.76 & 0.74 \\
\hline SP1A-18 & 0.85 & 1 & 70 & 0.76 & 0.76 \\
\hline SP1A 21 & 1 & 1 & 80 & 0.81 & 0.83 \\
\hline SP2A-13 & 0.955 & 2 & 50 & 0.57 & 0.59 \\
\hline SP2A-18 & 1.31 & 2 & 70 & 0.60 & 0.59 \\
\hline
\end{tabular}

The calculation results coincide quite well with the characteristics of the manufacturer and, therefore, the system of equations can be used as an energy model of a centrifugal well pump to calculate its energy efficiency. It should be borne in mind that the model is valid only when using the pump in continuous or intermittent modes. The use of frequency converters for capacity control leads to a change in pump performance and is not considered in this case.

Figure 2 shows the comparative results of calculations of specific energy consumption of a number of borehole pumps of the Grundfoss, Promelectro and Belamos trademarks, performed under the same initial conditions $(H=60 \mathrm{~m})$. For the comparison, we selected pumps, for which a sufficient set of source data is in free access. 


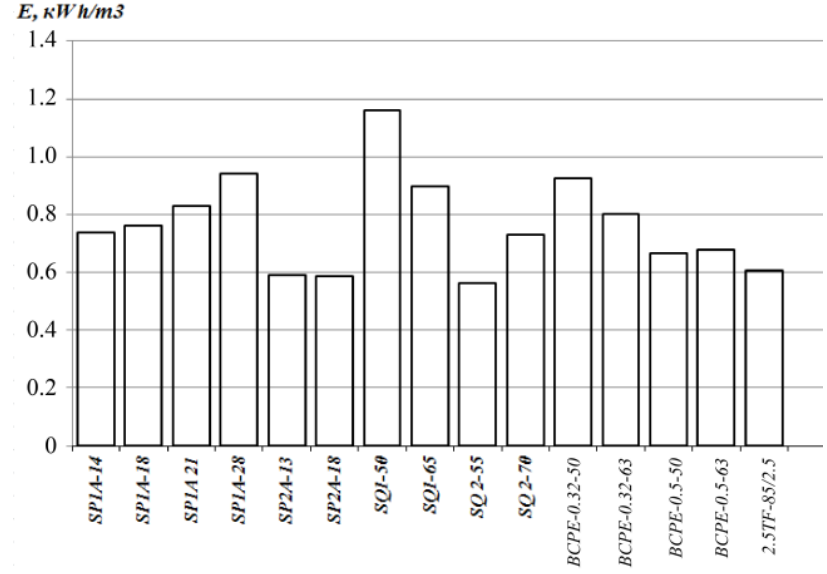

Fig. 2. Comparison of energy efficiency of products from different manufacturers.

The ratio of maximum and minimum energy efficiency indicators for the considered sampling is $1.16: 0.49=2.36$.

\section{Conclusions}

The conducted studies show that when choosing a centrifugal borehole pump for a water supply system, the cost-effectiveness criterion should not be only the product price. For making a final decision, energy consumption indicators must be taken into account. Otherwise, operating costs in the water supply system may be unnecessarily high.

\section{References}

[1] D. Borkowski, A. Wetula, and A. Bie, ISA Transactions 51(4), 539-549 (2012)

[2] E.P. Vetrov, Water and ecology: problems and solutions 1-2(42-43), 40-47 (2010)

[3] A.M. Vodovozov, N.N. Chernyaeva, SouthSiberian Scientific Bulletin 2(22), 10-152 (2018)

[4] E. Gruzdeva, Industrial control systems and controllers 5, 41-43 (2009)

[5] Ya.I. Maslova, and S.Z. Zhiznin, Economy and entrepreneurship 8-2(85), 623-627 (2017)

[6] S.V. Gordeev, Chief Power Engineer 1, 2931 (2010)

[7] V.I. Shcherbakov, and I.Yu. Purusova, Energy saving and water treatment 2(106), 27-32 (2017)

[8] O.A. Lysenko, Bulletin of the Tomsk Polytechnic University (Geo Assets Engineering. 325(4), 133-141 (2014)

[9] A.V. Kikot, and A.S. Levashov, Materials of the VI International Baltic Maritime Forum 342-347 (2018)

[10] A.M. Vodovozov, Automation and energy saving of machine-building and metallurgical production, technology and reliability of machines, devices and equipment 71-74 (2017)

[11] Grundfos Product Center. URL http://product-selection.grundfos.com
[12] I.I. Pavlinova, V.I. Bazhenov, I.G. Gubiy, Water supply and sanitation (Moscow: Yurayt) 380 (2017)

[13] I.V. Bochkarev, Problems of automation and control 1(32), 60-63 (2017) 